\title{
An Efficient 3d Head Pose Inference from Videos
}

\author{
Mohamed Dahmane and Jean Meunier \\ DIRO, Université de Montréal, CP 6128, Succursale Centre-Ville, \\ 2920 Chemin de la tour, Montréal, Québec, Canada, H3C 3J7 \\ \{dahmanem, meunier\}@iro. umontreal.ca
}

\begin{abstract}
In this article, we propose an approach to infer the 3d head pose from a monocular video sequence. First, we employ a Gabor-Phase based displacement estimation technique to track face features (two inner eye corners, two wings, tip and root of the nose). The proposed method is based on the iterative Lowe's pose estimation technique using the six tracked image facial points and their corresponding absolute location in a $3 \mathrm{~d}$ face model. As any iterative technique, the estimation process needs a good initial approximate solution that is found from orthography and scaling. With this method, the pose parameters are accurately obtained as continuous angular measurements rather than expressed in a few discrete orientations. Experimental results showed that under the assumption of a reasonable accuracy of facial features location, the method yields very satisfactory results.
\end{abstract}

Keywords: Head-pose estimation, facial features, face tracking, facial analysis.

\section{Introduction}

The orientation of the human head allows inferring important non-verbal forms of communication in a face-to-face talk (e.g. spatial information about the focus of attention, agreement-disagreement, confusion... ). The computer vision community is thus interested in automating interpersonal information captured from a scene. Thereby specific meaning can be automatically extracted from video by head pose inference, a process that presents an important challenge arising from individual appearance and personal facial dynamics.

The head pose can also be used to wrap and normalize the face for more general facial analysis, or in some augmented reality systems to construct images that wrap around the sides of the head.

For videos in which the face occupies less than 30 by 30 pixels (very low resolution), 3d model based approaches are inefficient [1]. The pose estimation problem is rather converted into a classical classification problem.

For somewhat higher resolution videos, since facial features remain hard to detect and track, adapted approaches were developed, such as appearance-based techniques (e.g. [2]).

In the case of high resolution face images, geometric-based approaches becomes relevant since facial features are visible and can be accurately recovered [3].

A. Elmoataz et al. (Eds.): ICISP 2010, LNCS 6134, pp. 368-375 2010.

(C) Springer-Verlag Berlin Heidelberg 2010 
In recent years, a variety of methods have been introduced, the reader is therefore invited to look at the thorough review presented in [4].

In this paper we deal with high resolution videos and use a geometric approach for head pose assessment since it can directly exploit properties that are known to influence human pose [4]. A further motivation behind using featurebased approaches is their near invariance under pose and orientation changes [5]. However, their performance depends on the precise configuration of the local facial features. Therefore, an effective scheme for tracking the features on the face would be essential to compensate for this shortcoming.

\section{Feature-Based Facial Tracking Scheme}

Generally, facial feature tracking methods, mainly based on Gabor wavelets, try to perform refinement stages [ 6 - 8] by imposing geometric constraints with subspace projection techniques or by using gray-level profiles to refine and adjust the positions of the features of interest. In this paper, we adopt a facial feature-based tracking using Gabor phase-based technique. In what follows, we propose to use a personalized gallery of facial bunch graphs (sets of Gabor jets attached to each node), that we deform to fit a set of tracked points using the Procrustes transform. Six particular facial feature points (left/right eye-inner corner, left/right nose wings, root and tip of the nose) are used because they are known to be less sensitive to facial deformations (Fig. 10.

\subsection{Procrustes Transform}

Procrustes shape analysis is a method in directional statistics [9], used to compare two shape configurations.

A two-dimensional shape can be described by a centered configurations $\mathbf{u}$ in $\mathcal{C}^{k}\left(\mathbf{u} \mathbf{1}_{k}=0\right)$, where $\mathbf{u}$ is a vector containing $2 \mathrm{~d}$ shape landmark points, each represented by a complex number of the form $x+\imath y$.

The procrustes transform is the similarity transform (eq. 1) that minimizes (eq. 2), where $\alpha \mathbf{1}_{k},|\beta|$ and $\angle \beta$, respectively, translates, scales and rotates $\mathbf{u}_{2}$, to match $\mathbf{u}_{1}$.

$$
\begin{gathered}
\left\{\begin{array}{c}
\mathbf{u}_{1}=\alpha \mathbf{1}_{k}+\beta \mathbf{u}_{2} \\
\beta=|\beta| e^{\imath \angle \beta}
\end{array} \quad \alpha, \beta \in \mathcal{C}\right. \\
\left\|\frac{\mathbf{u}_{1}}{\left\|\mathbf{u}_{1}\right\|}-\alpha \mathbf{1}_{k}-\beta \frac{\mathbf{u}_{2}}{\left\|\mathbf{u}_{2}\right\|}\right\|^{2}
\end{gathered}
$$

\subsection{Tracking of Facial Reference-Points}

From frame to frame, the tracking of the 4 reference points (left and right eye inner corner and the two nose wings) is based on an iterative disparity estimation procedure (Gabor phase-based technique described in [10]). To ensure a high 
tracking efficiency, we create a personnalized bunch for each one of these features. The size of each bunch depends on the degree of deformation of the corresponding feature.

\subsection{Geometric Fitting}

The Procrustes transform is used to adequately deform each reference graph $G_{i}$ stored in the personalized gallery. The transformation, that best wraps the 4 anchor points of $G_{i}$ to fit the tracked reference points (circle-dots in figure 1), is used to adjust the positions of the two remaining feature points of $G_{i}$ (diamonddots in figure 1). The new generated positions form the probe graph $G_{p}$.

\subsection{Refinement Stage}

Then, Gabor jets are calculated at each point position of the generated graph $G_{p}$, and a Jet-based similarity [10] is computed between each reference graph $G_{i}$ and the corresponding $G_{p}$. The probe graph with the highest similarity gives the positions of the six facial feature points. The final positions are obtained by estimating the optimal displacement of each point by using the Gabor phasebased displacement estimation technique [10].

\section{Extracting the Pose of the Face}

For recovering the pose of the face, we use the positions of the six facial feature points ( $L-R$ eye-inner corner, $L-R$ nose wings, the root and the tip of the nose), that are less sensitive to facial deformations. The pose can be estimated from the topography of the nose, using the volume generated by these points as it can be seen from the face model (Fig. 2).

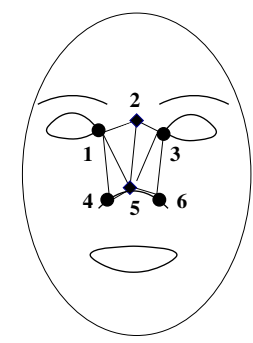

Fig. 1. The four tracked (circle) and the two adjusted (diamond) facial points

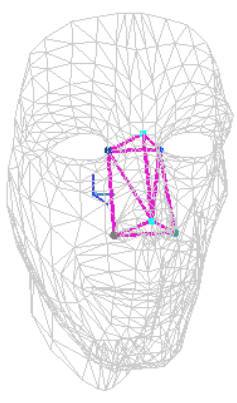

Fig. 2. The volume approaching the topography of the nose on a $3 d$ GeoFace model

Given an approximate 3d absolute position of each point of this volume and the corresponding $2 \mathrm{~d}$ points on the face image, we estimate the pose of the head using Lowe's pose estimation algorithm [11]. 


\subsection{Lowe's Pose Estimation Method}

Given $P_{i}(1 \leq i \leq n)$ the set of three-dimensional model points, expressed in the model reference frame, if $P_{i}^{\prime}$ represents the corresponding set, expressed in the camera reference frame, and if we denote by $p_{i}\left(x_{i}, y_{i}\right)$ the corresponding set of $2 \mathrm{~d}$ image points, the pose estimation problem is to solve for the parameter 1 $\mathbf{s}=(\mathbf{R}, \mathbf{T})$ so that

$$
\left[X_{i}^{\prime}, Y_{i}^{\prime}, Z_{i}^{\prime}\right]=\mathbf{R} P_{i}+\mathbf{T}
$$

where $p_{i}$ is the perspective projection of $P_{i}$

$$
\left[x_{i}, y_{i}\right]=f\left[\frac{X_{i}^{\prime}}{Z_{i}^{\prime}}, \frac{Y_{i}^{\prime}}{Z_{i}^{\prime}}\right]=\operatorname{Proj}\left(\tilde{\mathbf{s}}, P_{i}\right)
$$

For $\tilde{\mathbf{s}}$, an estimate of $\mathbf{s}$, an error measurement between the observations and the locations of $p_{i}$ is computed (eq. 5 ).

$$
\mathbf{e}_{i}=p_{i}-\operatorname{Proj}(\tilde{\mathbf{s}}, P)
$$

The pose parameters correction amount $\delta \mathbf{s}$, that eliminates the residual $\mathbf{e}$, can be found via the Newton Method's [12]. Lowe's method proceeds by producing new estimates for the parameters $\mathbf{s}$ (eq. 6) and iterating the procedure until the residual e (eq. 55) drops below a given tolerance.

$$
\mathbf{s}^{(i+1)}=\mathbf{s}^{(i)}+\delta \mathbf{s}
$$

\subsection{Initialization}

The iterative Newton's method starts off with an initial guess $\mathbf{s}^{(0)}$ which should be sufficiently accurate to ensure convergence to the true solution. As any iterative methods, choosing $\mathbf{s}^{(0)}$ from an appropriate well-behaved region is essential. For this purpose, we use the POS2 algorithm [13 15], which gives a reasonable rough estimate for $\mathbf{s}^{(0)}$. The algorithm approximates the perspective projection with a scaled orthographic projection, and finds the rotation matrix and the translation vector of the $3 \mathrm{~d}$ object by solving a linear system.

If $p$ and $P$ denote, respectively, the image points and the model points, the initial solution $\mathbf{s}^{(0)}$ can be determined, by recovering the rotation matrix $\mathbf{R}^{(0)}$ and the translation vector $\mathbf{T}^{(0)}$, from $P_{i} P_{1}$ and $p_{i} p_{1}$, two matrices constructed as follows,

$$
P_{i} P_{1}=\left(\begin{array}{ccc}
X_{2}-X_{1} & Y_{2}-Y_{1} & Z_{2}-Z_{1} \\
\vdots & \vdots & \vdots \\
X_{n}-X_{1} & Y_{n}-Y_{1} & Z_{n}-Z_{1}
\end{array}\right) \quad ; \quad p_{i} p_{1}=\left(\begin{array}{ccc}
x_{2}-x_{1} & y_{2}-y_{1} \\
\vdots & \vdots \\
x_{n}-x_{1} & y_{n}-y_{1}
\end{array}\right)
$$

\footnotetext{
${ }^{1} \mathbf{s}$ is the pose vector concatenating the three rotation angles (roll, pitch and yaw) and the $x, y, z$ translations.

2 Pose from Orthography and Scaling.
} 
The initial rotation matrix $\mathbf{R}^{(0)}$ is formed as

$$
\left(\begin{array}{c}
\mathbf{a}_{N}^{T} \\
\mathbf{b}_{N}^{T} \\
\left(\mathbf{a}_{N}^{T} \times \mathbf{b}_{N}^{T}\right)
\end{array}\right)
$$

where $(\mathbf{a}, \mathbf{b})$ is the matrix obtained by the left division $3 P_{i} P_{1} \backslash p_{i} p_{1}$, $\mathbf{a}$ and $\mathbf{b}$ are three-dimensional vectors. Subscript ${ }_{N}$ refers to the normalized vector and the symbol " $\times$ " to the cross product of two vectors.

The initial translation vector $\mathbf{T}^{(0)}$ is defined as

$$
\frac{\left(\bar{p}_{x}, \bar{p}_{y}, f\right)^{T}}{s c}
$$

where, $s c$ refers to the scale of the projection that corresponds to $(\|\mathbf{a}\|+\|\mathbf{b}\|) / 2$, $\bar{p}=\frac{1}{n} \sum p_{i}$, and $f$ is the camera focal length in pixels.

\section{Experimental Results}

To test the approach on real world data, we used representative video sequences displaying different head movements and orientations. In order to enhance the tracking performance, a personalized gallery was built with graph 4 (Fig. 1) from different faces under different "key orientations".

First, the subgraph containing the four reference facial features (Fig. 1) is roughly localized in the first frame of the video via an exhaustive search of the subgraph as a rigid object through the coarsest face image level. We used a three-level hierarchical image representation to decrease the inherent average latency of the graph search operation which is based on the Gabor jet similarity, by reducing the image search area and the size of the jet. For images at the finest level $(640 \times 480$ pixel resolution), jets are defined as sets of 40 complex coefficients constructed from different Gabor filters spanning 8 orientations under 5 scales. Whereas those for images at the coarsest level $(320 \times 240)$ are formed by 16 coefficients obtained from filters spanning 8 orientations under 2 scales. The intermediate level images use jets of $(8 \times 3)$ coefficients.

Then, the iterative displacement estimation procedure is used as a refinement stage, performed individually on each position of all of the six feature-points, and over the three levels of the pyramid face-image.

From frame to frame, only the four reference points are tracked using the iterative disparity estimation procedure. To avoid drifting during tracking, for each feature point, a local fitting is performed by searching through the gallery the subgraph that maximizes Gabor magnitude-based similarity. The rough

3 The left division $A \backslash B$ is the solution to the equation $A X=B$.

4 From our experiment, we found that about twenty graphs are sufficient to cover the different head appearances under various orientations. 
positions of the four feature points are given by the positions of the nodes of the optimal subgraph. These are then adjusted using the displacement estimation procedure.

The entire $3 d$ head pose inference method is summarized in the flow diagram of figure 3 .

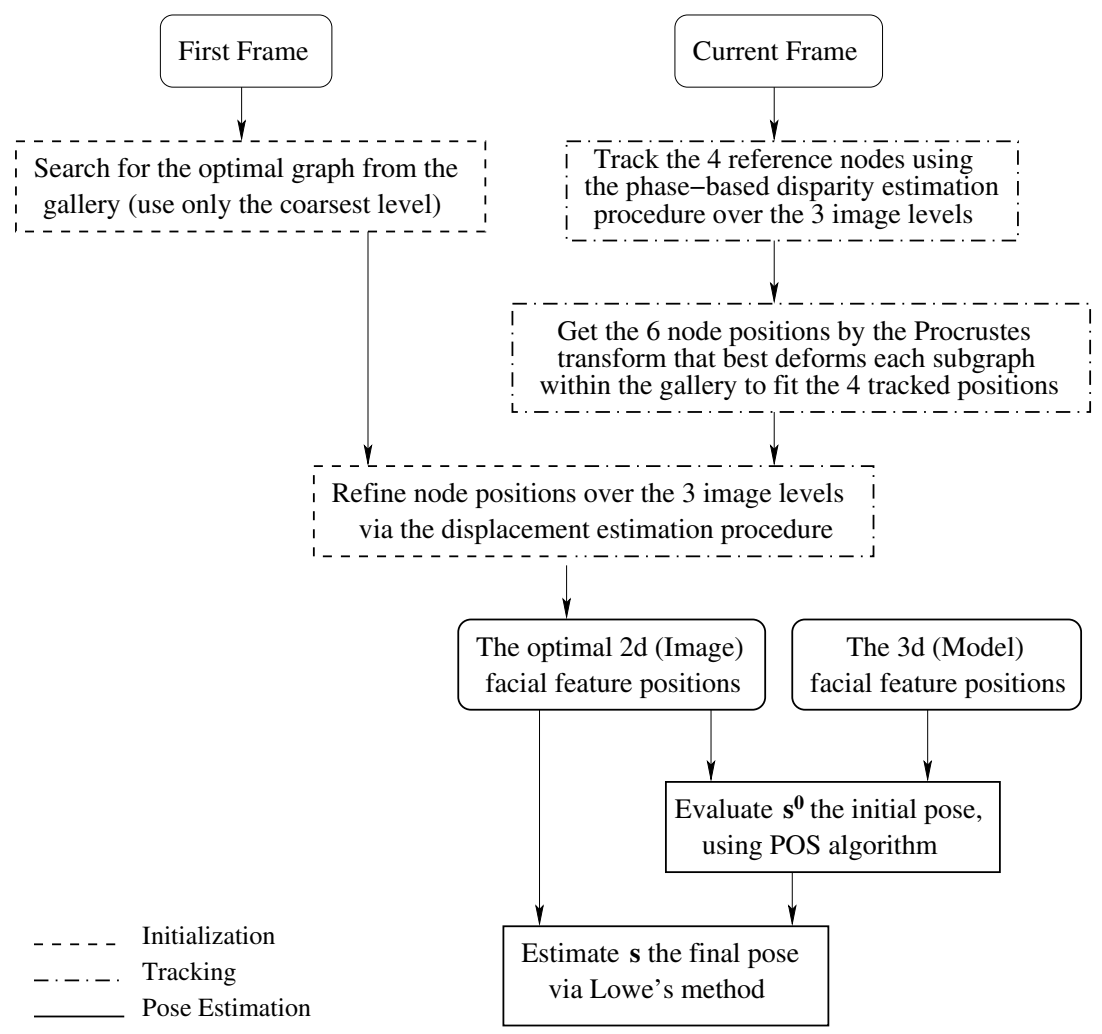

Fig. 3. Flow diagram of the entire $3 d$ pose inference approach

Figure 4 shows the tracking and pose inference results for 3 different persons and environment conditions.

Clearly, the achieved pose recovering performance are visually consistent with orientation of the head, as it is shown by the direction of the normal of the face shown in yellow color in figure 4 .

Figure 5 gives an example where a tracking failure occurred (see the tip of the nose). In this case a correcting mechanism (e.g. reinitialization based on poor Gabor similarity) can be adopted to prevent the tracker from drifting. 


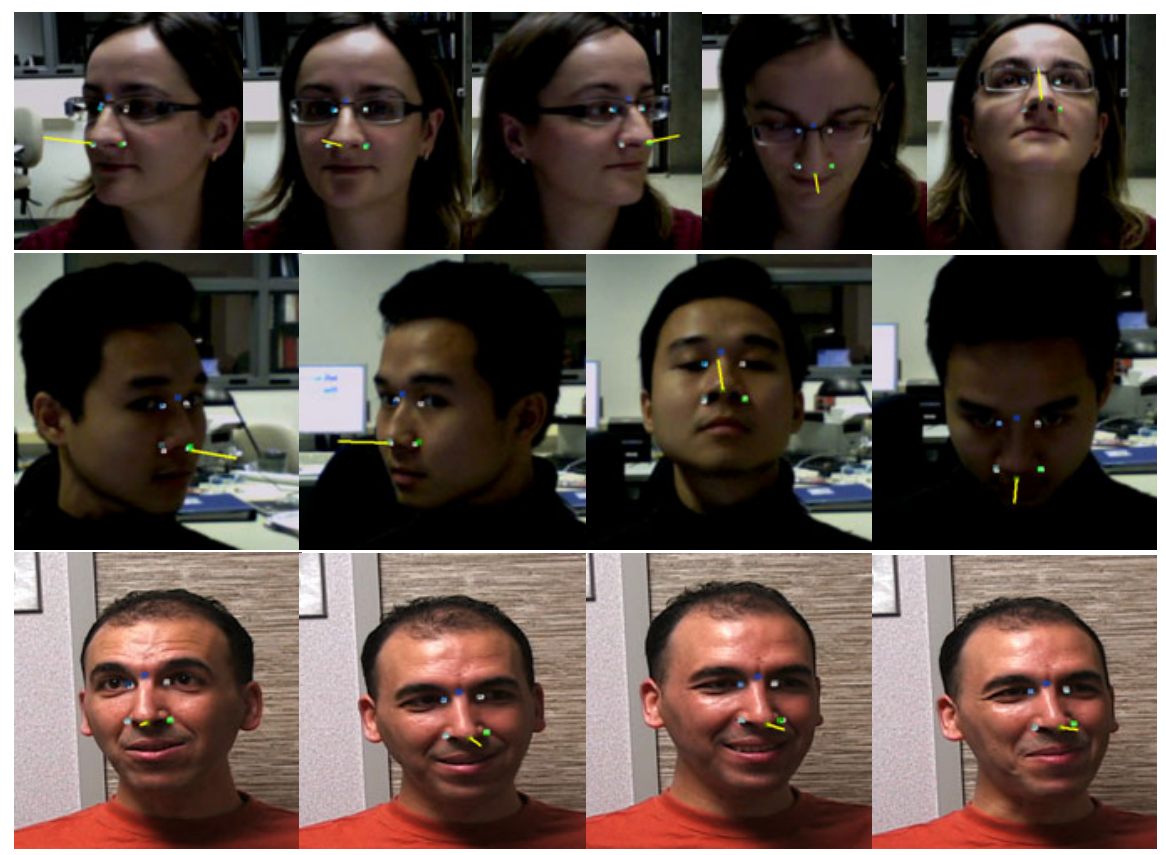

Fig. 4. Tracking and pose recovery performances

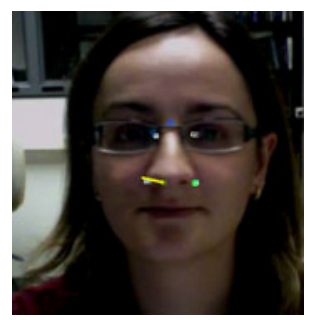

Fig. 5. A tracking failure of the nose tip affects the pose recovery

\section{Conclusions}

This article has described a feature-based pose estimation approach using the iterative Lowe's pose estimation technique, with six tracked image facial points and their corresponding locations in a $3 \mathrm{~d}$ face model. The estimated orientation parameters are given as continuous angular measurements rather than expressed in a few discrete orientations such as (left, up, front ...), furthermore the solution provides information about the $3 \mathrm{~d}$ position of the object. As any iterative solution, the estimation process needs an accurate initialization seed, which can be done using the pose from orthography and scaling algorithm. The facial features' tracking is accomplished by using the phase-based displacement 
estimation technique and a personalized gallery of facial bunch graphs to further enhance the tracking efficiency.

In the future, we plan to assess non-verbal communications between a patient and his health care professional in clinical setting.

\section{Acknowledgements}

This research was supported by the National Sciences and Engineering Research Council (NSERC) of Canada.

\section{References}

1. Jilin, T., Huang, T., Hai, T.: Accurate head pose tracking in low resolution video. In: 7th Int. Conf. on Automatic Face and Gesture Recognition, pp. 573-578 (2006)

2. Fu, Y., Huang, T.: Graph embedded analysis for head pose estimation. In: 7th International Conference on Automatic Face and Gesture Recognition (April 2006)

3. Hu, Y., Chen, L., Zhou, Y., Zhang, H.: Estimating face pose by facial asymmetry and geometry. In: Sixth IEEE International Conference on Automatic Face and Gesture Recognition, May 2004, pp. 651-656 (2004)

4. Murphy-Chutorian, E., Trivedi, M.: Head pose estimation in computer vision: A survey. IEEE Transactions on Pattern Analysis and Machine Intelligence 31(4), 607-626 (2009)

5. Yang, M.: Recent advances in face detection. In: Tutorial of IEEE Conferece on Pattern Recognition (2004)

6. McKenna, S.J., Gong, S., Würtz, R.P., Tanner, J., Banin, D.: Tracking facial feature points with gabor wavelets and shape models. In: Bigün, J., Borgefors, G., Chollet, G. (eds.) AVBPA 1997. LNCS, vol. 1206, pp. 35-42. Springer, Heidelberg (1997)

7. Zhu, Z., Ji, Q.: Robust pose invariant facial feature detection and tracking in realtime. In: 18th International Conference on Pattern Recognition, pp. 1092-1095 (2006)

8. Yu, M.S., Li, S.F.: Tracking facial feature points with statistical models and gabor wavelet. In: Fifth Mexican International Conference on Artificial Intelligence, November 2006, pp. 61-67 (2006)

9. Mardia, K., Jupp, P.: Directional Statistics. Wiley, New York (2000)

10. Dahmane, M., Meunier, J.: Constrained phase-based personalized facial feature tracking. In: Blanc-Talon, J., Bourennane, S., Philips, W., Popescu, D., Scheunders, P. (eds.) ACIVS 2008. LNCS, vol. 5259, pp. 1124-1134. Springer, Heidelberg (2008)

11. Lowe, D.G.: Fitting parametrized three-dimensional models to images 13(5), 441-450 (1991)

12. Trucco, E., Verri, A.: Introductory Techniques for 3-D Computer Vision, Englewood cliffs edn. Prentice-Hall, NJ (1998)

13. DeMenthon, D.F., Davis, L.S.: Model-based object pose in 25 lines of code. International Journal of Computer Vision 15, 123-141 (1995)

14. Tomasi, C., Kanade, T.: Shape and motion from image streams under orthography: a factorization method. Int. J. Comput. Vision 9(2), 137-154 (1992)

15. Ullman, S., Basri, R.: Recognition by linear combinations of models. IEEE Transactions on Pattern Analysis and Machine Intelligence 13(10), 992-1006 (1991) 